\title{
Association of surgeon and hospital volume with postoperative mortality after total gastrectomy for gastric cancer: data from 71,307 Japanese patients collected from a nationwide web-based data entry system
}

\author{
Masaaki Iwatsuki ${ }^{1} \cdot$ Hiroyuki Yamamoto ${ }^{2,3} \cdot$ Hiroaki Miyata ${ }^{2,3} \cdot$ Yoshihiro Kakeji $^{4} \cdot$ Kazuhiro Yoshida $^{5}$. \\ Hiroyuki Konno ${ }^{6} \cdot$ Yasuyuki Seto $^{7} \cdot \mathrm{Hideo} \mathrm{Baba}^{1} \mathbb{D}$
}

Received: 22 July 2020 / Accepted: 22 September 2020 / Published online: 9 October 2020

(c) The International Gastric Cancer Association and The Japanese Gastric Cancer Association 2020

\begin{abstract}
Background Despite interest in surgeon and hospital volume effects on total gastrectomy (TG), clinical significance has not been confirmed in a large-scale population. This study aimed at clarifying the association of surgeon and hospital volume on postoperative mortality after TG for gastric cancer among Japanese patients in National Clinical Database (NCD).

Methods Between 2011 and 2015, we retrospectively extracted data on TG for gastric cancer from the NCD. The primary outcome was operative mortality. We divided surgeon volume as the number of TGs performed by a patient's surgeon in the previous year: S1 (0-2 cases), S2 (3-9), S3 (10-25), S4 (26-79) and hospital volume by the number of TGs performed in the previous year: H1 (0-11 cases), H2 (12-26), H3 (27-146). We calculated the 95\% confidence interval (CI) for the mortality rate based on odds ratios (OR) estimated from a hierarchical logistic regression model.

Results We analyzed 71,307 patients at 2051 institutions. Low-volume surgeons and hospitals had significantly older and poorer-risk patients with various comorbidities. The operative mortality rate decreased with surgeon volume, $2.5 \%$ in $\mathrm{S} 1$ and $0.6 \%$ in $\mathrm{S} 4$. The operative mortality was $3.1 \%$ in $\mathrm{H} 1,1.7 \%$ in $\mathrm{H} 2$, and $1.2 \%$ in $\mathrm{H} 3$. After risk adjustment for surgeon, hospital volume and patient characteristics, hospital volume was significantly associated with operative morality (H3: OR $=0.53$, 95\% CI 0.43-0.63).

Conclusions We demonstrate hospital volume has an impact on postoperative mortality after TG in a nationwide population study. These findings suggest centralization may improve outcomes after TG.
\end{abstract}

Keywords Gastric cancer · Total gastrectomy $\cdot$ Surgeon volume $\cdot$ Hospital volume

\section{Introduction}

Gastric cancer is one of the most common cancers and is associated with a high mortality rate worldwide [1]. The prognosis of patients with advanced gastric cancer remains

Hideo Baba

hdobaba@kumamoto-u.ac.jp

1 Department of Gastroenterological Surgery, Graduate School of Medical Sciences, Kumamoto University, 1-1-1 Honjo, Chuo-ku, Kumamoto 860-8556, Japan

2 Department of Healthcare Quality Assessment, Graduate School of Medicine, The University of Tokyo, Tokyo, Japan

3 Department of Health Policy and Management, School of Medicine, Keio University, Tokyo, Japan poor despite of the recent progress in cancer treatment. Gastrectomy with regional lymph node dissection is the most effective treatment for gastric cancer. For advanced gastric cancer, gastrectomy with D2 lymph node dissection is recommended worldwide [2-4]. Though subtotal gastrectomy is

4 Database Committee, The Japanese Society of Gastroenterological Surgery, Tokyo, Japan

5 Department of Surgical Oncology, Gifu University School of Medicine, Gifu, Japan

6 Hamamatsu University School of Medicine Hamamatsu, Hamamatsu, Japan

7 The Japanese Society of Gastroenterological Surgery, Tokyo, Japan 
the preferred surgical procedure whenever possible in order to limit post-operative morbidity and to optimize quality of life, total gastrectomy (TG) is indicated in the treatment of certain gastric cancers, including that located in the upper third of the stomach or advanced gastric cancer extending to the cardia, diffuse signet ring gastric adenocarcinoma, and hereditary diffuse gastric cancer (CDH1 mutation), both in the prophylactic setting.

TG is well known as complex procedure that carries a significant risk of postoperative morbidity and mortality. Major complications after TG including esophagojejunal anastomotic leakage, duodenal stump leakage, and pancreatic fistula related to lymphadenectomy can be fatal. Serious morbidity and mortality were significantly higher in the TG group than the partial gastrectomy group [5]. Large Western centers have previously demonstrated the data regarding morbidity and mortality after TG, which conclude the 30-day morbidity and mortality rates of approximately $30 \%$ and 5\%, respectively [5, 6]. In Eastern, Watanabe et al. reported that the overall morbidity was $26.2 \%$, with a 30 -day mortality rate of $0.9 \%$ based on the National Clinical Database (NCD) and established risk stratification model of postoperative outcomes and identification of patients at risk for morbidity [7].

Recently, there is a growing recognition that the multidisciplinary care in high-volume hospital can improve postoperative both short and long outcomes of gastric cancer patients [8,9]. Claassen et al. demonstrated that gastric surgery performed in high-volume hospital was associated with better surgical quality and prognosis based on data from CRITICS trial [10, 11]. In Germany, the observational study using national hospital discharge data reveled that treatment in very high volume is associated with lower mortality compared to low-volume hospital [12]. In also Eastern, hospital volume-outcome relationship was demonstrated for perioperative mortality after TG using Taiwan National Insurance Research Database between 2000 and 2010 [13]. However, the relationship between surgeon and hospital volume with postoperative morbidity and mortality after TG for gastric cancer remains unknown in a larger and more recently cohort.

In Japan, NCD was founded in 2010 as the parent body of the database system linked to the board certification system, which contains records of $\geq 95 \%$ of the surgeries performed by regular surgeons in Japan [14]. Almost 5000 facilities have enrolled, and over 11,300,000 cases have been registered. Most recently, we revealed that hospital volume had a strong impact on postoperative mortality after distal gastrectomy (DG) for Japanese patients with gastric cancer using NCD [15]. In the current study, to clarify the impact of surgeon and hospital volume on postoperative mortality for TG, we evaluated data from 71,307 Japanese patients with gastric cancer enrolled in NCD.

\section{Methods}

\section{Data collection}

From 2011, the NCD collected data on more than $11,300,000$ surgical cases from more than 5000 institutes. In the gastroenterological surgery section, the database registered all surgical cases that fell into this category; in addition, it required detailed input items for eight procedures, including gastrectomy, that were determined to represent the performance of surgery in each specialty. The NCD constructed software for an Internet-based data collection system, and the data managers of the participating hospitals were responsible for forwarding their data to the NCD office. The NCD ensures traceability of its data by maintaining continuity in the staff who approve the data, the staff of the departments in charge of annual cases, and the data-entry personnel. It also validates data consistency via random inspections of participating institutions.

In this study, we focused on the specific NCD section for gastrointestinal surgery. Briefly, potential independent variables included patient demographics, pre-existing comorbidities, preoperative laboratory values, and operative data.

\section{Patients}

A total of 71,307 patients, who underwent TG for gastric cancer at 2051 institutions between January 1, 2011 and December 31, 2015, were eligible for analysis. Records with missing data on patient age, sex, or outcome were excluded.

\section{Endpoint}

The primary outcome measure of this study was 30-day and operative mortalities. Operative mortality was defined as death during the index hospitalization, regardless of the length of hospital stay ( $\leq 90$ days), as well as after hospital discharge within 30 days from the operation date.

\section{Surgeon and hospital volume}

We defined surgeon volume as the number of TGs performed by a patient's surgeon in the previous year. Surgeon volume was divided into the following four groups: S1 ( $0-2$ cases per year), S2 (3-9 cases), S3 (10-25 cases), and $\mathrm{S} 4(26+$ cases $)$. We divided hospital volume by the number of TGs performed in the previous year into the following three tertiles: H1 (0-11 cases per year), H2 (12-26 cases), and H3 (27-146). 


\section{Statistical methods}

All statistical calculations were performed with STATA 15 (STATA Corp., TX, USA). We compared median values with the Kruskal-Wallis test for operation time and estimated blood loss, and Chi-squared test for all other variables. All $P$ values were two-sided, and we considered $P<0.05$ as statistically significant. First, we analyzed the relationship between annual surgical volume (hospital volume or surgeon volume) and surgical mortality after gastrectomy with hierarchical logistic regression models accounting for clustering of patients by surgeons and hospital levels. To adjust for patient-level risk factors, the following variables, which were used in the scoring system established by NCD data [16], were utilized: demographic factors, such as age category; preoperative functional status, such as need for any assistance with activities of daily living (ADL); history of cerebrovascular disease; weight loss more than $10 \%$; uncontrolled ascites; ASA score class 3 or more; pre-existing comorbidities, such as the presence of respiratory distress, disseminated cancer, operative factors, such as emergency surgery, with pancreatectomy and laparoscopic gastrectomy; and preoperative laboratory data, such as white blood cell count more than $11,000 / \mu \mathrm{L}$, anemia (hematocrit $<30 \%$ ), serum albumin less than $3.0 \mathrm{~g} / \mathrm{dL}$, alkaline phosphatase more than $600 \mathrm{IU} / \mathrm{L}$, serum Na less than $138 \mathrm{mEq} / \mathrm{L}$, and prothrombin time-international normalized ratio more than 1.25 , low platelet count $\left(<12 \times 10^{4} / \mu \mathrm{L}\right)$, aspartate aminotransferase more than $35 \mathrm{IU} / \mathrm{L}$, and increased level of total bilirubin ( $>2 \mathrm{mg} / \mathrm{dL})$. In addition, to illustrate the relationship between operative mortality and surgeon volume as a continuous variable, generalized estimation equation logistic regression models were utilized, in which a restricted cubic spline model was implemented. All procedures were conducted in accordance with the ethical standards of the respective committees on human experimentation (institutional and national) and with the Helsinki Declaration. An ethics committee that includes members of the Japanese Surgical Society ethics board, lawyers, patient representatives, and experts on information security that considered the ethical propriety of the entire initiative approved it and made the review process public on the Japan Surgical Society website. The use of data from the registry for retrospective observational studies was approved by The Japanese Society of Gastroenterological Surgery committee and the institutional Review Board of Kumamoto University committee (No. 1514), and individual written or verbal informed consent was waived because of the retrospective design.

\section{Results}

We retrieved data on a total of 71,307 patients who underwent TG for gastric cancer by 10,274 surgeons at 2051 institutions from January 2011 to December 2015. Surgeon volume ranged from 1 to 79 TG per year. Annual surgeon volume was divided into the following four groups: S1: 0-2 $(n=34,362), \mathrm{S} 2: 3-9(n=27,532), \mathrm{S} 3: 10-25(n=8088)$, and S3: 26-79 $(n=1325)$ cases per year (Fig. 1a).

Patient demographic data and preoperative risk assessment according to surgeon volume are summarized in Table 1. Low-volume surgeons had operated on significantly older patients and poorer-risk patients with various comorbidities and organ dysfunctions. More laparoscopic a

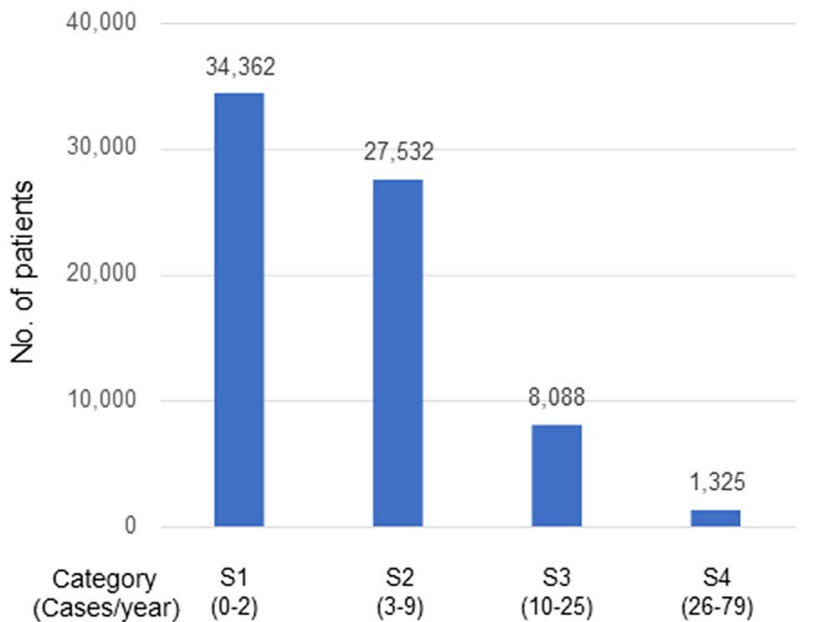

b

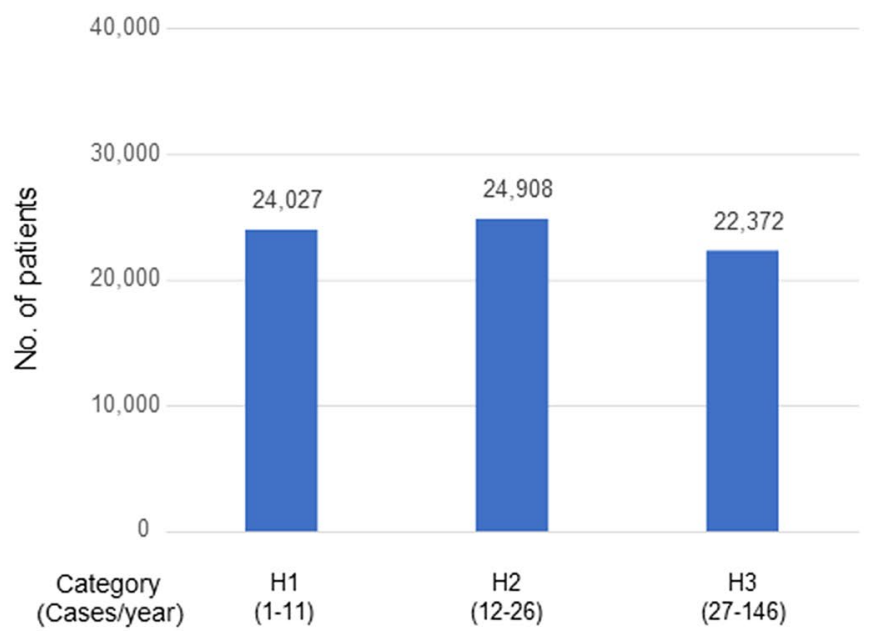

Fig. 1 The definition and distribution of surgeon volume and hospital volume a surgeon volume, b hospital volume 
Table 1 Preoperative risk assessment and surgical outcomes according to surgeon volume category

\begin{tabular}{|c|c|c|c|c|c|c|c|c|c|c|c|c|}
\hline \multirow[t]{2}{*}{ Variables } & \multicolumn{2}{|c|}{$\begin{array}{l}\mathrm{S} 1(0-2 \\
\text { cases }) \\
(n=34,362)\end{array}$} & \multicolumn{2}{|c|}{$\begin{array}{l}\mathrm{S} 2(3-9 \\
\text { cases }) \\
(n=27,532)\end{array}$} & \multicolumn{2}{|c|}{$\begin{array}{l}\mathrm{S} 3(10-25 \\
\text { cases }) \\
(n=8088)\end{array}$} & \multicolumn{2}{|c|}{$\begin{array}{l}\mathrm{S} 4(26-79 \\
\text { cases }) \\
(n=1325)\end{array}$} & \multicolumn{2}{|c|}{$\begin{array}{l}\text { Total } \\
(n=71,307)\end{array}$} & \multirow[t]{2}{*}{ Pearson Chi-square } & \multirow[t]{2}{*}{$P$ value } \\
\hline & No. & $\%$ & No. & $\%$ & No. & $\%$ & No. & $\%$ & No. & $\%$ & & \\
\hline \multicolumn{13}{|l|}{ Demographics } \\
\hline Age & & & & & & & & & & & 419.518 & $<0.001$ \\
\hline 59 & 4443 & 12.9 & 3904 & 14.2 & 1480 & 18.3 & 299 & 22.6 & 10,126 & 14.2 & & \\
\hline $60-64$ & 4299 & 12.5 & 3681 & 13.4 & 1118 & 13.8 & 219 & 16.5 & 9317 & 13.1 & & \\
\hline $65-69$ & 5765 & 16.8 & 4763 & 17.3 & 1465 & 18.1 & 233 & 17.6 & 12,226 & 17.1 & & \\
\hline $70-74$ & 6882 & 20.0 & 5493 & 20.0 & 1595 & 19.7 & 233 & 17.6 & 14,203 & 19.9 & & \\
\hline $75-79$ & 6655 & 19.4 & 5158 & 18.7 & 1389 & 17.2 & 195 & 14.7 & 13,397 & 18.8 & & \\
\hline 80 & 6318 & 18.4 & 4533 & 16.5 & 1041 & 12.9 & 146 & 11.0 & 12,038 & 16.9 & & \\
\hline \multicolumn{13}{|l|}{ Preoperative risk assessment } \\
\hline Respiratory distress & 785 & 2.3 & 530 & 1.9 & 94 & 1.2 & 14 & 1.1 & 1423 & 2.0 & 50.061 & $<0.001$ \\
\hline Preoperative ADL: any assistance & 1748 & 5.1 & 1220 & 4.4 & 212 & 2.6 & 15 & 1.1 & 3195 & 4.5 & 129.734 & $<0.001$ \\
\hline Ascites without control & 839 & 2.4 & 613 & 2.2 & 144 & 1.8 & 14 & 1.1 & 1610 & 2.3 & 22.400 & $<0.001$ \\
\hline Preoperative dialysis & 217 & 0.6 & 171 & 0.6 & 27 & 0.3 & 3 & 0.2 & 418 & 0.6 & 13.569 & 0.004 \\
\hline Previous cerebrovascular disease & 688 & 2.0 & 523 & 1.9 & 95 & 1.2 & 13 & 1.0 & 1319 & 1.8 & 30.591 & $<0.001$ \\
\hline Disseminated cancer & 957 & 2.8 & 683 & 2.5 & 132 & 1.6 & 17 & 1.3 & 1789 & 2.5 & 44.368 & $<0.001$ \\
\hline Weight loss $>10 \%$ & 3135 & 9.1 & 2069 & 7.5 & 429 & 5.3 & 63 & 4.8 & 5696 & 8.0 & 166.769 & $<0.001$ \\
\hline ASA $\geq$ grade 3 & 3696 & 10.8 & 3068 & 11.1 & 746 & 9.2 & 94 & 7.1 & 7604 & 10.7 & 42.286 & $<0.001$ \\
\hline \multicolumn{13}{|l|}{ Preoperative laboratory data } \\
\hline Hematocrit $<30 \%$ & 4772 & 13.9 & 3568 & 13.0 & 789 & 9.8 & 111 & 8.4 & 9240 & 13.0 & 124.525 & $<0.001$ \\
\hline Serum albumin $<3.0 \mathrm{~g} / \mathrm{dL}$ & 3315 & 9.6 & 2232 & 8.1 & 445 & 5.5 & 43 & 3.2 & 6035 & 8.5 & 204.811 & $<0.001$ \\
\hline Total bilirubin $>2 \mathrm{mg} / \mathrm{dL}$ & 232 & 0.7 & 153 & 0.6 & 39 & 0.5 & 5 & 0.4 & 429 & 0.6 & 7.121 & 0.068 \\
\hline AST $>35 \mathrm{IU} / \mathrm{L}$ & 2580 & 7.5 & 2026 & 7.4 & 542 & 6.7 & 76 & 5.7 & 5224 & 7.3 & 11.309 & 0.01 \\
\hline $\mathrm{ALP}>600 \mathrm{IU} / \mathrm{L}$ & 278 & 0.8 & 213 & 0.8 & 52 & 0.6 & 2 & 0.2 & 545 & 0.8 & 9.081 & 0.028 \\
\hline $\mathrm{Na}<138 \mathrm{mEq} / \mathrm{L}$ & 2956 & 8.6 & 2307 & 8.4 & 551 & 6.8 & 70 & 5.3 & 5884 & 8.3 & 43.729 & 0.001 \\
\hline PT-INR > 1.25 & 918 & 2.7 & 689 & 2.5 & 153 & 1.9 & 16 & 1.2 & 1776 & 2.5 & 25.576 & $<0.001$ \\
\hline White blood cells $>11,000 / \mathrm{mL}$ & 1122 & 3.3 & 759 & 2.8 & 169 & 2.1 & 29 & 2.2 & 2079 & 2.9 & 39.266 & $<0.001$ \\
\hline \multicolumn{13}{|l|}{ Surgical factors } \\
\hline Laparoscopic approach & 4063 & 11.8 & 5946 & 21.6 & 2993 & 37.0 & 405 & 30.6 & 13,407 & 18.8 & 3112.393 & $<0.001$ \\
\hline With pancreatectomy & 600 & 1.7 & 540 & 2.0 & 161 & 2.0 & 23 & 1.7 & 1324 & 1.9 & 4.863 & 0.182 \\
\hline Emergent surgery & 436 & 1.3 & 326 & 1.2 & 69 & 0.9 & 6 & 0.5 & 837 & 1.2 & 15.808 & 0.001 \\
\hline \multicolumn{13}{|l|}{ Postoperative outcomes } \\
\hline \multicolumn{13}{|l|}{ Surgical complications } \\
\hline Operative mortality & 850 & 2.5 & 487 & 1.8 & 96 & 1.2 & 8 & 0.6 & 1441 & 2.0 & 86.258 & $<0.001$ \\
\hline Surgical site infection & 1765 & 5.1 & 1410 & 5.1 & 422 & 5.2 & 66 & 5.0 & 3663 & 5.1 & 0.188 & 0.980 \\
\hline Anastomotic leakage & 1640 & 4.8 & 1321 & 4.8 & 325 & 4.0 & 53 & 4.0 & 3339 & 4.7 & 10.827 & 0.013 \\
\hline Pancreatic fistula & 1543 & 4.5 & 1371 & 5.0 & 463 & 5.7 & 79 & 6.0 & 3456 & 4.8 & 27.603 & $<0.001$ \\
\hline \multicolumn{13}{|l|}{ Nonsurgical complications } \\
\hline Pneumonia & 1285 & 3.7 & 954 & 3.5 & 227 & 2.8 & 29 & 2.2 & 2495 & 3.5 & 24.205 & $<0.001$ \\
\hline Acute renal failure & 436 & 1.3 & 263 & 1.0 & 50 & 0.6 & 7 & 0.5 & 756 & 1.1 & 35.788 & $<0.001$ \\
\hline Reoperation within 30 days & 1904 & 5.5 & 1447 & 5.3 & 368 & 4.5 & 44 & 3.3 & 3763 & 5.3 & 23.513 & $<0.001$ \\
\hline Unplanned intubation & 581 & 1.7 & 412 & 1.5 & 83 & 1.0 & 6 & 0.5 & 1082 & 1.5 & 30.103 & $<0.001$ \\
\hline Cardiac events & 202 & 0.6 & 127 & 0.5 & 32 & 0.4 & 3 & 0.2 & 364 & 0.5 & 9.569 & 0.023 \\
\hline Septic shock & 992 & 2.9 & 709 & 2.6 & 165 & 2.0 & 14 & 1.1 & 1880 & 2.6 & 32.890 & $<0.001$ \\
\hline Transfusion & 459 & 1.3 & 313 & 1.1 & 75 & 0.9 & 6 & 0.5 & 853 & 1.2 & 17.627 & 0.001 \\
\hline Events in central nervous system & 102 & 0.3 & 88 & 0.3 & 18 & 0.2 & 1 & 0.1 & 209 & 0.3 & 4.204 & 0.24 \\
\hline Prolonged ventilation $>48 \mathrm{~h}$ & 618 & 1.8 & 424 & 1.5 & 85 & 1.1 & 6 & 0.5 & 1133 & 1.6 & 35.981 & $<0.001$ \\
\hline
\end{tabular}


TG were performed by the high-volume surgeons $(P<0.001)$. The operative mortality rate decreased with surgeon volume, $2.5 \%$ in $\mathrm{S} 1,1.8 \%$ in $\mathrm{S} 2,1.2 \%$ in $\mathrm{S} 3$, and $0.6 \%$ in S4. Regarding surgical complications, anastomotic leakage was more frequently observed among low-volume surgeons and the incidence of pancreatic fistula was higher among high-volume surgeons $(P<0.001)$, which is consistent with our DG data [15]. The rate of all nonsurgical complications including pneumonia, the reoperation rate and the rate of septic shock were significantly higher among low-volume surgeons $(P<0.001)$.

Hospital volume ranged from 1 to $146 \mathrm{TG}$ per year. Annual hospital volume was distributed among the tertiles as follows: category H1: $1-11(n=24,027), \mathrm{H} 2$ : 12-26 $(n=24,908)$, and H3: 27-146 $(n=22,327)$ cases per year (Fig. 1b). Patient demographic data and preoperative risk assessment according to hospital volume category are summarized in Table 2. Low-volume hospitals had significantly older patients and poorer-risk patients with various comorbidities and organ dysfunctions. More laparoscopic TG were performed in the high-volume hospitals $(P<0.001)$. Operative mortality was $3.1 \%$ in $\mathrm{H} 1$, $1.7 \%$ in $\mathrm{H} 2$, and $1.2 \%$ in $\mathrm{H} 3$. A significant reduction in mortality rate was observed according to hospital volume $(P<0.001)$. Regarding surgical complications, we found no significant difference of the rate of anastomotic leakage between in low- and high-volume hospitals. The incidence of pancreatic fistula was higher in high-volume hospitals, which is similar to low-volume surgeons $(P<0.001)$. The rate of all nonsurgical complications excluding pneumonia, the rate of septic shock, and event rate of central nervous system were significantly higher in low-volume hospitals $(P<0.001)$.

Table 3 summarizes the $95 \%$ CIs for overall mortality after TG from the hierarchical logistic regression models. The lowest volume surgeons were significantly associated with higher mortality (Fig. 2a, S1: OR, 1.29, 95\% CI 1.01-1.64, $P=0.043$ ), and higher-volume hospitals were significantly associated with a decreased risk of mortality in a dose-dependent manner (Fig. 2b, H2: OR, 0.61, 95\% CI $0.53-0.71 ; P<0.001, \mathrm{H} 3: \mathrm{OR}, 0.51,95 \%$ CI $0.43-0.61$, $P<0.001)$. After risk adjustment for surgeon and hospital volume, hospital volume was significantly associated with operative morality (Fig. 2d, H2: OR, 0.63, 95\% CI $0.54-0.73, P<0.001$; H3: OR, $0.53,95 \%$ CI $0.43-0.63$, $P<0.001$ ), whereas surgeon volume was not (Fig. 2c), which is consistent with our DG data [15].

Furthermore, the OR for operative mortality gradually decreased in a surgeon volume-dependent manner after risk adjustment for both only patient factors such as demographic factors, preoperative functional status, pre-existing comorbidities, operative factors, and preoperative laboratory data (Fig. 3a) and adding hospital volume (Fig. 3b). OR for operative mortality reaches the plateau in about 40 cases/ year with or without risk adjustment.

\section{Discussion}

In the current nationwide study, we revealed that hospital volume was associated with postoperative mortality after TG compared to surgeon volume among 71,307 Japanese patients with gastric cancer. This result is consistent with our previous data regarding DG and Taiwan study by $\mathrm{Wu}$ et al. $[13,15]$. Recently, there are growing evidences that hospital volume is associated with postoperative mortality based on national database and large-scale clinical trials [10, 11]. However, most of studies included several types of gastrectomy such as TG and DG with or without the resection of the surrounding organs. To the best of our knowledge, this is the first largest scale of report focused on TG, which is the most complex procedure for gastric cancer using national clinical database.

$\mathrm{Wu}$ et al. first revealed the impact of the hospital volume of TG on the long-term surgical outcomes in 7905 patients with gastric cancer between 2000 and 2010 in nationwide study [13]. As Wu et al. discuss, this finding is attributed to some possible factors as follows: advances of perioperative care, more experienced surgical skills, and development of surgical devices in recent time period. On the issue of survey period, we believe that our data collected from NCD between 2011 and 2015 were more feasible for clinical practice in this era. Most recently, based on the data from CRITICS trial, pivotal clinical trials regarding perioperative adjuvant therapy for advanced gastric cancer conducted in Netherland, association between hospital volume and both short-, and long-term outcomes was published [10, 11]. Intriguingly, although there were no significant difference in postoperative complications or mortality between the hospital volume categories [11], high hospital volume was associated with higher overall and disease free survival [10]. Approximately 85\% hospital in the CRITICS trial has more than 20 resections per year. Therefore, centralizing gastrectomy for gastric cancer naturally occurs. However, it remains unknown whether centralization of gastric cancer surgery can improve morbidity and mortality rates in clinical practice because the patients enrolled into clinical trials are likely to have fair condition.

Previous studies that examined the centralization of gastric cancer treatment produced heterogeneous and conflicting findings due to technical, regional, and demographic factors. Nelen et al. recently reported that centralizing gastrectomy improved the number of harvested lymph nodes and successfully introduced laparoscopic gastrectomy [8]. Furthermore, Lee et al. demonstrated that hospital volume did not directly affect postoperative morbidity and mortality 
Table 2 Preoperative risk assessment and surgical outcomes according to hospital volume category

\begin{tabular}{|c|c|c|c|c|c|c|c|c|c|c|}
\hline \multirow[t]{2}{*}{ Variables } & \multicolumn{2}{|c|}{$\begin{array}{l}\text { H1 }(1-11 \\
\text { cases }) \\
(n=24,027)\end{array}$} & \multicolumn{2}{|c|}{$\begin{array}{l}\mathrm{H} 2(12-26 \\
\text { cases }) \\
(n=24,908)\end{array}$} & \multicolumn{2}{|c|}{$\begin{array}{l}\text { H3 }(27-146 \\
\text { cases) } \\
(n=22,372)\end{array}$} & \multicolumn{2}{|c|}{$\begin{array}{l}\text { Total } \\
(n=71,307)\end{array}$} & \multirow[t]{2}{*}{ Pearson Chi-square } & \multirow[t]{2}{*}{$P$ value } \\
\hline & No. & $\%$ & No. & $\%$ & No. & $\%$ & No. & $\%$ & & \\
\hline \multicolumn{11}{|l|}{ Demographics } \\
\hline Age & & & & & & & & & 619.651 & $<0.001$ \\
\hline 59 & 2909 & 12.1 & 3398 & 13.6 & 3819 & 17.1 & 10,126 & 14.2 & & \\
\hline $60-64$ & 2957 & 12.3 & 3200 & 12.8 & 3160 & 14.1 & 9317 & 13.1 & & \\
\hline $65-69$ & 3904 & 16.2 & 4285 & 17.2 & 4037 & 18.0 & 12,226 & 17.1 & & \\
\hline $70-74$ & 4633 & 19.3 & 5138 & 20.6 & 4432 & 19.8 & 14,203 & 19.9 & & \\
\hline $75-79$ & 4750 & 19.8 & 4775 & 19.2 & 3872 & 17.3 & 13,397 & 18.8 & & \\
\hline 80 & 4874 & 20.3 & 4112 & 16.5 & 3052 & 13.6 & 12,038 & 16.9 & & \\
\hline \multicolumn{11}{|l|}{ Preoperative risk assessment } \\
\hline Respiratory distress & 676 & 2.8 & 447 & 1.8 & 300 & 1.3 & 1423 & 2.0 & 136.350 & $<0.001$ \\
\hline Preoperative ADL: any assistance & 1423 & 5.9 & 1143 & 4.6 & 629 & 2.8 & 3195 & 4.5 & 263.019 & $<0.001$ \\
\hline Ascites without control & 597 & 2.5 & 554 & 2.2 & 459 & 2.1 & 1610 & 2.3 & 10.040 & 0.007 \\
\hline Preoperative dialysis & 162 & 0.7 & 143 & 0.6 & 113 & 0.5 & 418 & 0.6 & 5.784 & 0.055 \\
\hline Previous cerebrovascular disease & 541 & 2.3 & 482 & 1.9 & 296 & 1.3 & 1319 & 1.8 & 56.555 & $<0.001$ \\
\hline Disseminated cancer & 796 & 3.3 & 615 & 2.5 & 378 & 1.7 & 1789 & 2.5 & 125.062 & $<0.001$ \\
\hline Weight loss $>10 \%$ & 2520 & 10.5 & 1866 & 7.5 & 1310 & 5.9 & 5696 & 8.0 & 351.114 & $<0.001$ \\
\hline ASA $\geq$ grade 3 & 2584 & 10.8 & 2906 & 11.7 & 2114 & 9.4 & 7604 & 10.7 & 61.156 & $<0.001$ \\
\hline \multicolumn{11}{|l|}{ Preoperative laboratory data } \\
\hline Hematocrit $<30 \%$ & 3514 & 14.6 & 3286 & 13.2 & 2440 & 10.9 & 9240 & 13.0 & 143.908 & $<0.001$ \\
\hline Serum albumin $<3.0 \mathrm{~g} / \mathrm{dL}$ & 2502 & 10.4 & 2124 & 8.5 & 1409 & 6.3 & 6035 & 8.5 & 372.764 & $<0.001$ \\
\hline Total bilirubin $>2 \mathrm{mg} / \mathrm{dL}$ & 176 & 0.7 & 146 & 0.6 & 107 & 0.5 & 429 & 0.6 & 12.675 & 0.002 \\
\hline AST $>35 \mathrm{IU} / \mathrm{L}$ & 1831 & 7.6 & 1867 & 7.5 & 1526 & 6.8 & 5224 & 7.3 & 12.529 & 0.002 \\
\hline $\mathrm{ALP}>600 \mathrm{IU} / \mathrm{L}$ & 202 & 0.8 & 212 & 0.9 & 131 & 0.6 & 545 & 0.8 & 13.750 & 0.001 \\
\hline $\mathrm{Na}<138 \mathrm{mEq} / \mathrm{L}$ & 1987 & 8.3 & 2162 & 8.7 & 1735 & 7.8 & 5884 & 8.3 & 13.328 & 0.001 \\
\hline PT-INR $>1.25$ & 671 & 2.8 & 669 & 2.7 & 436 & 1.9 & 1776 & 2.5 & 39.975 & $<0.001$ \\
\hline White blood cells $>11,000 / \mathrm{mL}$ & 853 & 3.6 & 699 & 2.8 & 527 & 2.4 & 2079 & 2.9 & 60.016 & $<0.001$ \\
\hline \multicolumn{11}{|l|}{ Surgical factors } \\
\hline Laparoscopic approach & 3258 & 13.6 & 4632 & 18.6 & 5517 & 24.7 & 13,407 & 18.8 & 936.115 & $<0.001$ \\
\hline With pancreatectomy & 374 & 1.6 & 486 & 2.0 & 464 & 2.1 & 1324 & 1.9 & 18.894 & $<0.001$ \\
\hline Emergent surgery & 273 & 1.1 & 306 & 1.2 & 258 & 1.2 & 837 & 1.2 & 1.017 & 0.601 \\
\hline \multicolumn{11}{|l|}{ Postoperative outcomes } \\
\hline \multicolumn{11}{|l|}{ Surgical complications } \\
\hline Operative mortality & 745 & 3.1 & 429 & 1.7 & 267 & 1.2 & 1441 & 2.0 & 230.056 & $<0.001$ \\
\hline Surgical site infection & 1127 & 4.7 & 1328 & 5.3 & 1208 & 5.4 & 3663 & 5.1 & 14.929 & 0.001 \\
\hline Anastomotic leakage & 1130 & 4.7 & 1134 & 4.6 & 1075 & 4.8 & 3339 & 4.7 & 1.716 & 0.424 \\
\hline Pancreatic fistula & 821 & 3.4 & 1244 & 5.0 & 1391 & 6.2 & 3456 & 4.8 & 198.842 & $<0.001$ \\
\hline \multicolumn{11}{|l|}{ Nonsurgical complications } \\
\hline Pneumonia & 881 & 3.7 & 856 & 3.4 & 758 & 3.4 & 2495 & 3.5 & 3.102 & 0.212 \\
\hline Acute renal failure & 311 & 1.3 & 275 & 1.1 & 170 & 0.8 & 756 & 1.1 & 32.254 & $<0.001$ \\
\hline Reoperation within 30 days & 1416 & 5.9 & 1300 & 5.2 & 1047 & 4.7 & 3763 & 5.3 & 34.381 & $<0.001$ \\
\hline Unplanned intubation & 449 & 1.9 & 338 & 1.4 & 295 & 1.3 & 1082 & 1.5 & 30.051 & $<0.001$ \\
\hline Cardiac events & 174 & 0.7 & 108 & 0.4 & 82 & 0.4 & 364 & 0.5 & 33.634 & $<0.001$ \\
\hline Septic shock & 664 & 2.8 & 670 & 2.7 & 546 & 2.4 & 1880 & 2.6 & 5.134 & 0.077 \\
\hline Transfusion & 334 & 1.4 & 284 & 1.1 & 235 & 1.1 & 853 & 1.2 & 12.327 & 0.002 \\
\hline Events in central nervous system & 76 & 0.3 & 82 & 0.3 & 51 & 0.2 & 209 & 0.3 & 4.802 & 0.091 \\
\hline Prolonged ventilation $>48 \mathrm{~h}$ & 456 & 1.9 & 367 & 1.5 & 310 & 1.4 & 1133 & 1.6 & 22.702 & $<0.001$ \\
\hline
\end{tabular}


Table 3 Ninety-five percentage confidence intervals for overall mortality after total gastrectomy from the hierarchical logistic regression models

\begin{tabular}{|c|c|c|c|c|c|c|}
\hline & \multicolumn{2}{|l|}{ Surgeon volume } & \multicolumn{2}{|l|}{ Hospital volume } & \multicolumn{2}{|c|}{ Surgeon and hospital } \\
\hline & Odds ratio & $P$ value & Odds ratio & $P$ value & Odds ratio & $P$ value \\
\hline \multicolumn{7}{|c|}{ Surgeon volume } \\
\hline S1 & $1.29(1.01-1.64)$ & 0.043 & - & - & $1.00(0.77-1.29)$ & 0.976 \\
\hline S2 & $1.03(0.80-1.32)$ & 0.802 & - & - & $0.89(0.69-1.14)$ & 0.352 \\
\hline S3 & Ref & & - & - & Ref & \\
\hline S4 & $0.67(0.30-1.52)$ & 0.338 & - & - & $0.73(0.32-1.65)$ & 0.451 \\
\hline \multicolumn{7}{|c|}{ Hospital volume } \\
\hline H1 & - & - & Ref & & Ref & \\
\hline $\mathrm{H} 2$ & - & - & $0.61(0.53-0.71)$ & $<0.001$ & $0.63(0.54-0.73)$ & $<0.001$ \\
\hline H3 & - & - & $0.51(0.43-0.61)$ & $<0.001$ & $0.53(0.43-0.63)$ & $<0.001$ \\
\hline
\end{tabular}
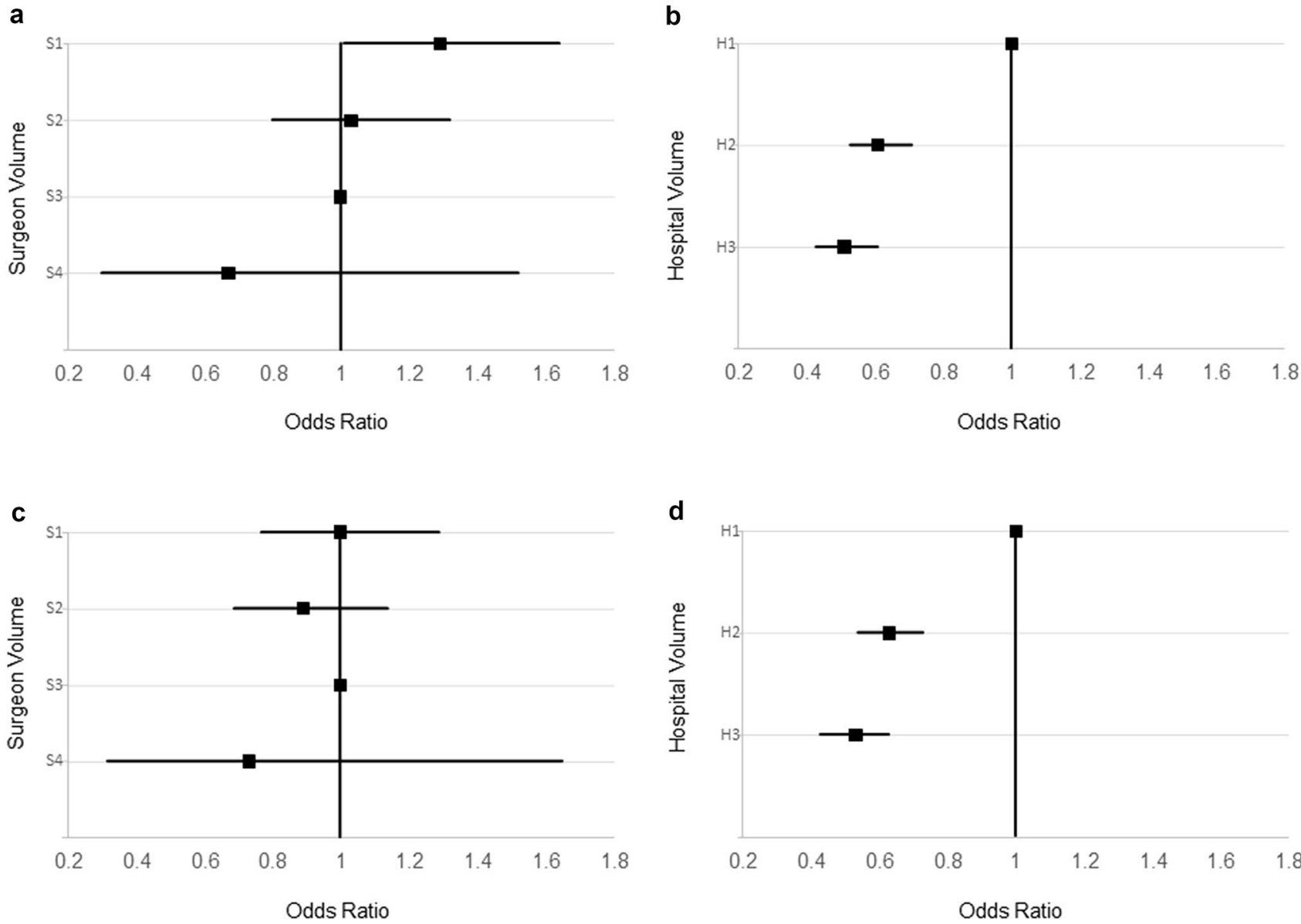

Fig. 2 Forest plot for overall mortality calculated by multivariate logistic regression analysis. a Surgeon volume adjusted by risk model variables, b hospital volume adjusted by risk model variables, c, d surgeon and hospital volume adjusted by risk model variables includ-

achieved by well-trained beginners of laparoscopic gastrectomy [17]. This finding suggests that surgeon volume is an important factor affecting postoperative outcomes in laparoscopic gastrectomy. On the other hand, Nelen et al. also showed that centralization of gastrectomy did not improve

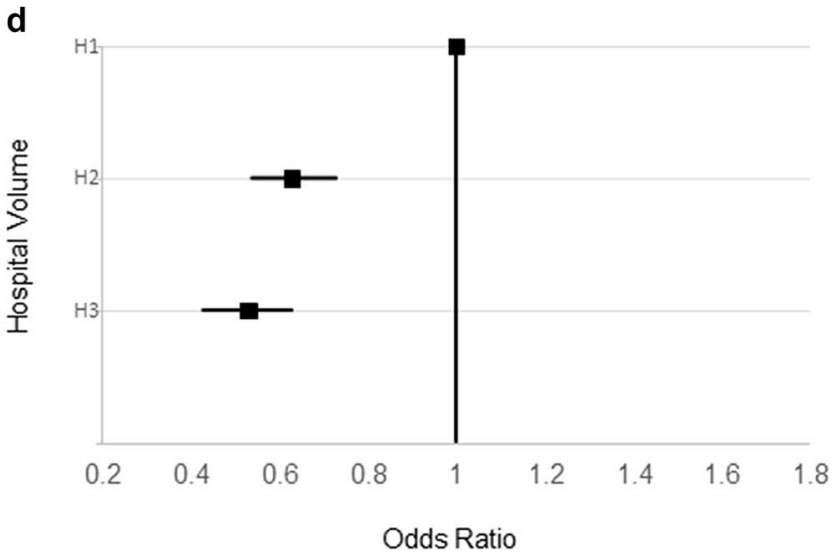

ing hospital volume. Demographic factors, preoperative functional status, pre-existing comorbidities, operative factors, and preoperative laboratory data weere utilized to adjust for patient-level risk factors

the mortality rate in the Eastern part of the Netherlands [8], which is similar to the results reported by Thompson et al. [18]. However, these studies were conducted in small cohorts and in some parts of the West. In Japan, morbidity and mortality rates of gastric cancer surgery are substantially 
a

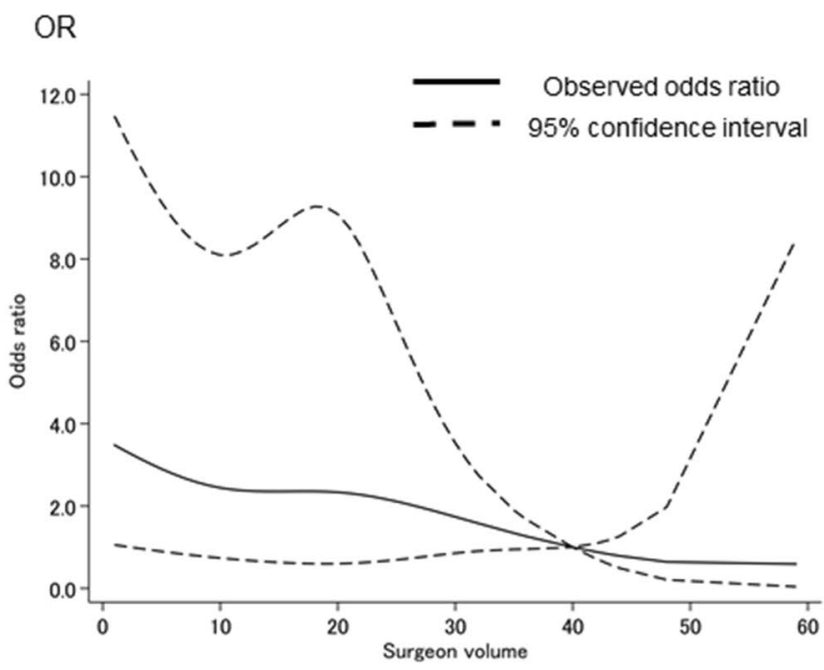

Fig. 3 Odds ratio of mortality after gastrectomy according to surgeon caseload per year $\mathbf{a}$ adjusted by risk model variables and $\mathbf{b}$ adjusted by risk model variables including hospital volume. Solid lines: observed odds ratio; dashed lined: $95 \%$ confidence interval from the

lower, even in with low surgeon and hospital volumes, compared to the West [19]. These findings lead to more questions as to how a low surgeon volume in a high-volume hospital can impact mortality.

There are several potential benefits for short-term outcomes that result from a high hospital volume. First, the surgeon can provide referrals to various experts for patients with postoperative morbidities. Second, high-volume hospitals generally have a sufficient cooperative structure for diagnostic and interventional procedures after postoperative morbidities. Third, surgeon volume will increase in highvolume centers under supervision by experts for gastrectomy, leading to lower mortality. Konno et al. reported that the participation of board-certified surgeons in gastroenterological surgery, including TG, contributes to favorable surgical outcomes of gastroenterological surgery using NCD data [20]. These potential benefits may lead to favorable short-term outcomes in higher volume hospitals. Furthermore, it is possible that the patient characteristics depend on each surgeon and hospital. Busweiler et al. reported that elderly patients might benefit specifically from centralization [21]. In this study, low-volume surgeons and hospitals had significantly older patients and poorer-risk patients with various comorbidities and organ dysfunctions (Tables 1,2). Our study is real-world evidence from a nationwide cohort study after adjustment for these independent variables for operative mortality, which is used in the scoring system established by the NCD data.

Our study has some limitations. First, long-term outcomes, such as recurrence-free survival and overall b

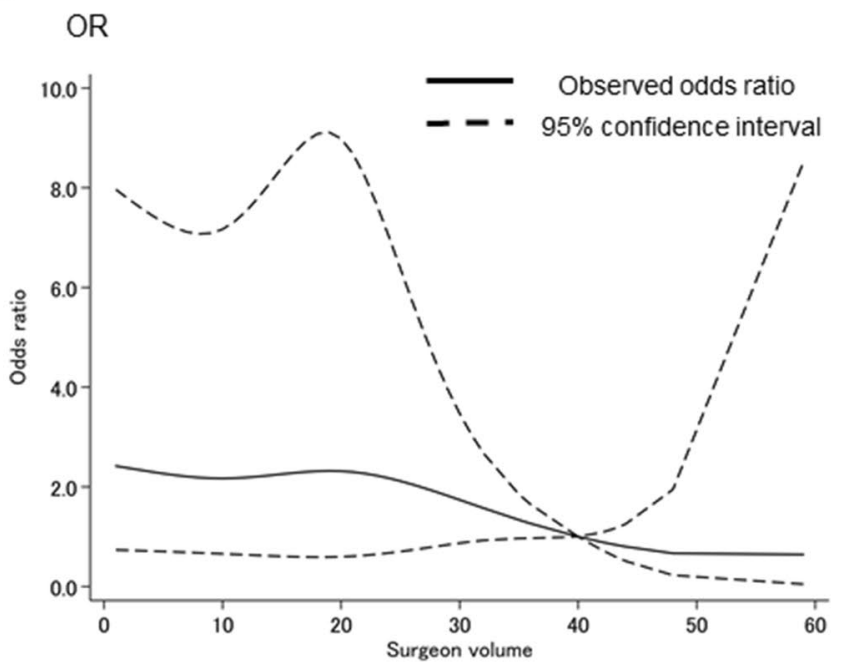

logistic regression model. Demographic factors, preoperative functional status, pre-existing comorbidities, operative factors, and preoperative laboratory data were utilized to adjust for patient-level risk factors

survival, were not evaluated. Further analysis of the impact of surgeon and hospital volume on long-term outcomes after TG is required, because postoperative complications can lead to adverse effects on OS and DFS [22, 23]. In Japan, the data of long-term outcome are interlinked to NCD by the nation gastric cancer registration system by Japanese Gastric Cancer Association. Second, it remains controversial whether the cut-off value of surgeon and hospital volume defined by Japanese population is appropriate to apply to clinical practice in other Eastern and Western countries. To clarify the criteria of the surgeon volume as a continuous variable, we analyzed OR of mortality by generalized estimation equation logistic regression models. The OR of mortality according to surgeon caseload per year reaches the plateau in about 40 cases per year. However, it is difficult for a surgeon to experience 40 cases per year even if in high-volume hospitals. Furthermore, it is possible that surgeon volume drastically changes every year, depending on hospital where surgeon belongs, whereas hospital volume is assumed to be constant. This is possible reason why hospital volume had a strong impact on postoperative mortality after TG. Therefore, to determine the concrete threshold of surgeon and hospital volume, the differences of epidemiology, biology, and treatment strategy of each country should be considered. Despite these limitations, it is possible that our results have implications for improving healthcare delivery. 


\section{Conclusions}

Hospital volume had a strong impact on postoperative mortality after TG for Japanese patients with gastric cancer in a nationwide web-based data entry system, NCD, which suggests that centralization may improve outcomes after TG.

Acknowledgements The authors thank all of the data managers and hospitals participating in this NCD project for their great efforts in entering the data analyzed in this study. The authors also thank Prof Hideki Hashimoto and Noboru Motomura, MD, for providing direction for the foundation of the NCD and the working members of the JSGS database committee (Harushi Udagawa, MD; Michiaki Unno, MD; Itaru Endo, MD; Chikara Kunisaki, MD; Akinobu Taketomi, MD; Akira Tangoku, MD; Tadahiko Masaki, MD; Shigeru Maruhashi, MD; Kazuhiro Yoshida, MD). The authors also thank Hiroyuki Konno, MD, for his important suggestions regarding this article.

\section{Compliance with ethical standards}

Conflict of interest Hiroaki Miyata and Hiroyuki Yamamoto are affiliated with the Department of Healthcare Quality Assessment at the University of Tokyo. The department is a social collaboration department supported by grants from the National Clinical Database, Johnson \& Johnson K.K., and Nipro Co. None of these organizations had any role in the design and conduct of the study, data collection, data analysis, data management, data interpretation, or the preparation, review, and approval of this manuscript. This work was supported in part by a Grant-in-Aid for Scientific Research from the Japan Society for the Promotion of Science, grant number 20K07594 (For M.I.).

\section{References}

1. Siegel RL, Miller KD, Jemal A. Cancer statistics 2020. CA Cancer J Clin. 2020;70(1):7-30.

2. Ajani JA, D’Amico TA, Almhanna K, Bentrem DJ, Chao J, Das $\mathrm{P}$, et al. Gastric cancer, version 3.2016, NCCN clinical practice guidelines in oncology. J Natl Compr Cancer Netw JNCCN. 2016;14(10):1286-312.

3. Japanese Gastric Cancer A. Japanese gastric cancer treatment guidelines 2014 (ver. 4). Gastric Cancer. 2017;20(1):1-19.

4. Smyth EC, Verheij M, Allum W, Cunningham D, Cervantes A, Arnold D, et al. Gastric cancer: ESMO Clinical Practice Guidelines for diagnosis, treatment and follow-up. Ann Oncol. 2016;27(suppl 5):v38-v49.

5. Papenfuss WA, Kukar M, Oxenberg J, Attwood K, Nurkin S, Malhotra U, et al. Morbidity and mortality associated with gastrectomy for gastric cancer. Ann Surg Oncol. 2014;21(9):3008-144.

6. Bartlett EK, Roses RE, Kelz RR, Drebin JA, Fraker DL, Karakousis GC. Morbidity and mortality after total gastrectomy for gastric malignancy using the American College of Surgeons National Surgical Quality Improvement Program database. Surgery. 2014;156(2):298-304.

7. Watanabe M, Miyata H, Gotoh M, Baba H, Kimura W, Tomita $\mathrm{N}$, et al. Total gastrectomy risk model: data from 20,011 Japanese patients in a nationwide internet-based database. Ann Surg. 2014;260(6):1034-9.

8. Nelen SD, Heuthorst L, Verhoeven RHA, Polat F, Kruyt PM, Reijnders $\mathrm{K}$, et al. Impact of centralizing gastric cancer surgery on treatment, morbidity, and mortality. J Gastrointest Surg. 2017;21(12):2000-8.
9. van Putten M, Nelen SD, Lemmens V, Stoot J, Hartgrink HH, Gisbertz SS, et al. Overall survival before and after centralization of gastric cancer surgery in the Netherlands. Br J Surg. 2018;105(13):1807-15.

10. Claassen YHM, van Amelsfoort RM, Hartgrink HH, Dikken JL, de Steur WO, van Sandick JW, et al. Effect of hospital volume with respect to performing gastric cancer resection on recurrence and survival: results from the CRITICS trial. Ann Surg. 2019;270(6):1096-102.

11. Claassen YHM, van Sandick JW, Hartgrink HH, Dikken JL, De Steur WO, van Grieken NCT, et al. Association between hospital volume and quality of gastric cancer surgery in the CRITICS trial. Br J Surg. 2018;105(6):728-35.

12. Nimptsch U, Haist T, Gockel I, Mansky T, Lorenz D. Complex gastric surgery in Germany-is centralization beneficial? Observational study using national hospital discharge data. Langenbeck's Arch Surg. 2019;404(1):93-101.

13. Wu JM, Ho TW, Tien YW. Correlation between the increased hospital volume and decreased overall perioperative mortality in one universal health care system. World J Surg. 2019;43(9):2194-202.

14. Miyata H, Gotoh M, Hashimoto H, Motomura N, Murakami A, Tomotaki A, et al. Challenges and prospects of a clinical database linked to the board certification system. Surg Today. 2014;44(11):1991-9.

15. Iwatsuki M, Yamamoto H, Miyata H, Kakeji Y, Yoshida K, Konno $\mathrm{H}$, et al. Effect of hospital and surgeon volume on postoperative outcomes after distal gastrectomy for gastric cancer based on data from 145,523 Japanese patients collected from a nationwide webbased data entry system. Gastric Cancer. 2019;22(1):190-201.

16. Kurita N, Miyata H, Gotoh M, Shimada M, Imura S, Kimura $\mathrm{W}$, et al. Risk model for distal gastrectomy when treating gastric cancer on the basis of data from 33,917 Japanese patients collected using a nationwide web-based data entry system. Ann Surg. 2015;262(2):295-303.

17. Lee HH, Son SY, Lee JH, Kim MG, Hur H, Park DJ. Surgeon's experience overrides the effect of hospital volume for postoperative outcomes of laparoscopic surgery in gastric cancer: multiinstitutional study. Ann Surg Oncol. 2017;24(4):1010-7.

18. Thompson AM, Rapson T, Gilbert FJ, Park KG, Scottish Audit of $\mathrm{G}$, Oesophageal C. Hospital volume does not influence long-term survival of patients undergoing surgery for oesophageal or gastric cancer. Br J Surg. 2007;94(5):578-84.

19. Mamidanna R, Ni Z, Anderson O, Spiegelhalter SD, Bottle A, Aylin P, et al. Surgeon volume and cancer esophagectomy, gastrectomy, and pancreatectomy: a population-based study in England. Ann Surg. 2016;263(4):727-32.

20. Konno H, Kamiya K, Kikuchi H, Miyata H, Hirahara N, Gotoh $\mathrm{M}$, et al. Association between the participation of board-certified surgeons in gastroenterological surgery and operative mortality after eight gastroenterological procedures. Surg Today. 2017;47(5):611-8.

21. Busweiler LAD, Dikken JL, Henneman D, van Berge Henegouwen MI, Ho VKY, Tollenaar R, et al. The influence of a composite hospital volume on outcomes for gastric cancer surgery: a Dutch population-based study. J Surg Oncol. 2017;115(6):738-45.

22. Kubota T, Hiki N, Sano T, Nomura S, Nunobe S, Kumagai K, et al. Prognostic significance of complications after curative surgery for gastric cancer. Ann Surg Oncol. 2014;21(3):891-8.

23. Tokunaga M, Tanizawa Y, Bando E, Kawamura T, Terashima M. Poor survival rate in patients with postoperative intra-abdominal infectious complications following curative gastrectomy for gastric cancer. Ann Surg Oncol. 2013;20(5):1575-83.

Publisher's Note Springer Nature remains neutral with regard to jurisdictional claims in published maps and institutional affiliations. 